\title{
A Secure Multiparty Product Protocol for Preserving the Privacy in Collaborative Data Mining
}

\author{
G.Chitra Ganapathi \\ Lecturer, Department of CSE \\ V.L.B Janakiammal College of Engg \\ \&Tech \\ Coimbatore 42
}

\author{
G.Swathi \\ III CSE \\ V.L.B Janakiammal College of Engg
\&Tech \\ Coimbatore 42
}

\author{
S.Karthick \\ III CSE \\ V.L.B Janakiammal College of Engg \\ \&Tech \\ Coimbatore 42
}

\begin{abstract}
In the modern business world, collaborative data mining becomes especially important because of the mutual benefit it brings to the collaborators. During the collaboration, each party of the collaboration needs to share its data with other parties. If the parties don't care about their data privacy, the collaboration can be easily achieved. Privacy concerns parties, each having a private data set, want to jointly conduct association rule mining without disclosing their private data to other parties. This paper deals with how to conduct collaborative data mining, one of the core data mining techniques, on private data. There is no central, trusted party having access to all the data. Instead, a protocol using Homomorphic encryption-techniques, to exchange the data while keeping it private, is used.
\end{abstract}

The full text of the article is not available in the cache. Kindly refer the IJCA digital library at www.ijcaonline.org for the complete article. In case, you face problems while downloading the full-text, please send a mail to editor at editor@ijcaonline.org 